\title{
Monitoring dopants by Raman scattering in an electrochemically top-gated graphene transistor
}

\author{
A. DAS ${ }^{1}$, S. PISANA², B. CHAKRABORTY1 ${ }^{1}$, S. PISCANEC ${ }^{2}$, S. K. SAHA ${ }^{1}$, U. V. WAGHMARE ${ }^{3}$, \\ K. S. NOVOSELOV ${ }^{4}$, H. R. KRISHNAMURTHY ${ }^{1}$, A. K. GEIM ${ }^{4}$, A. C. FERRAR ${ }^{2 *}$ AND A. K. SOOD ${ }^{1 *}$ \\ 'Department of Physics, Indian Institute of Science, Bangalore 560012, India \\ ${ }^{2}$ Department of Engineering, Cambridge University, 9 JJ Thomson Avenue, Cambridge CB3 OFA, UK \\ ${ }^{3}$ Theoretical Sciences Unit, Jawaharlal Nehru Centre for Advanced Scientific Research, Bangalore 560064, India \\ ${ }^{4}$ Department of Physics and Astronomy, Manchester University, Manchester M13 9PL, UK \\ *e-mail: acf26@eng.cam.ac.uk; asood@physics.iisc.ernet.in
}

The recent discovery of graphene ${ }^{1-3}$ has led to many advances in two-dimensional physics and devices ${ }^{4,5}$. The graphene devices fabricated so far have relied on $\mathrm{SiO}_{2}$ back gating ${ }^{1-3}$. Electrochemical top gating is widely used for polymer transistors ${ }^{6,7}$, and has also been successfully applied to carbon nanotubes ${ }^{8,9}$. Here we demonstrate a top-gated graphene transistor that is able to reach doping levels of up to $5 \times 10^{13} \mathrm{~cm}^{-2}$, which is much higher than those previously reported. Such high doping levels are possible because the nanometre-thick Debye layer ${ }^{8,10}$ in the solid polymer electrolyte gate provides a much higher gate capacitance than the commonly used $\mathrm{SiO}_{2}$ back gate, which is usually about $300 \mathrm{~nm}$ thick ${ }^{11}$. In situ Raman measurements monitor the doping. The $G$ peak stiffens and sharpens for both electron and hole doping, but the $2 \mathrm{D}$ peak shows a different response to holes and electrons. The ratio of the intensities of the $G$ and 2D peaks shows a strong dependence on doping, making it a sensitive parameter to monitor the doping.

Figure 1a shows a schematic diagram of our experimental setup for transport and Raman measurements. (See Supplementary Information and Methods for details about device fabrication and measurements.) Figure $1 \mathrm{~b}$ shows the source-drain current $\left(I_{\mathrm{SD}}\right)$ of the top-gated graphene as a function of electrochemical gate voltage. The gate dependence of the drain current (Fig. 1b) shows ambipolar behaviour and is almost symmetric for both electron and hole doping. This is directly related to the band structure of graphene, where both electron and hole conduction are accessible by shifting the Fermi level. The $I_{\mathrm{SD}}-V_{\mathrm{DS}}$ characteristics at different electrochemical gate voltages (Fig. 1c) show linear behaviour, indicating the lack of significant Schottky barriers at the electrode-graphene interface.

In order to compare our top-gating results with the usual backgating measurements, it is necessary to convert the top-gate voltage into an effective doping concentration. In general, the application of a gate voltage $\left(V_{\mathrm{G}}\right)$ creates an electrostatic potential difference $\phi$ between the graphene and the gate electrode, and the addition of charge carriers leads to a shift in the Fermi level $\left(E_{\mathrm{F}}\right)$.
Therefore, $V_{\mathrm{G}}$ is given by

$$
V_{\mathrm{G}}=\frac{E_{\mathrm{F}}}{e}+\phi
$$

with $E_{\mathrm{F}} / e$ being determined by the chemical (quantum) capacitance of the graphene, and $\phi$ being determined by the geometrical capacitance $C_{\mathrm{G}}$. As discussed in the Methods section, for the back gate, $\phi \gg E_{\mathrm{F}} / e$, whereas for top gating the two terms in equation (1) are comparable.

The Fermi energy in graphene changes as $E_{F}(n)=\hbar\left|v_{F}\right| \sqrt{\pi n}$, where $\left|v_{\mathrm{F}}\right|=1.1 \times 10^{6} \mathrm{~ms}^{-1}$ is the Fermi velocity ${ }^{2,3}$. For the top gate, $\phi=n e / C_{\mathrm{TG}}$, where $C_{\mathrm{TG}}$ is the geometric capacitance (TG denotes 'top gate'). From equation (1) we get

$$
V_{\mathrm{TG}}=\frac{\hbar\left|v_{\mathrm{F}}\right| \sqrt{\pi n}}{e}+\frac{n e}{C_{\mathrm{TG}}}
$$

Using the numerical values: $C_{\mathrm{TG}}=2.2 \times 10^{-6} \mathrm{~F} \mathrm{~cm}^{-2}$ (as given in the Methods section) and $v_{\mathrm{F}}=1.1 \times 10^{6} \mathrm{~ms}^{-1}$,

$$
V_{\mathrm{TG}}(\text { volts })=1.16 \times 10^{-7} \sqrt{n}+0.723 \times 10^{-13} n
$$

where $n$ is in units of $\mathrm{cm}^{-2}$. Equation (3) allows us to estimate the doping concentration at each top-gate voltage $\left(V_{\mathrm{TG}}\right)$. Note that, as in back gating, we also obtain the minimum source-drain current at finite top-gate voltage $\left(V_{n \mathrm{TG}}=0.6 \mathrm{~V}\right)$, as seen in Fig. $1 \mathrm{~b}$. Accordingly, a positive (negative) $V_{\mathrm{TG}}-V_{n \mathrm{TG}}$ induces electron (holes) doping.

Figure $2 \mathrm{a}$ plots the resistivity of our graphene layer (extracted from Fig. 1b knowing the sample's aspect ratio: $W / L=1.55)$ as a function $V_{\mathrm{TG}}$. Figure $2 \mathrm{~b}$ shows the back-gate response of the same sample (without electrolyte). There is an increase in resistivity maximum $(\sim 6 \mathrm{k} \Omega)$ after pouring the electrolyte, which may originate from the creation of more charged impurities on the 

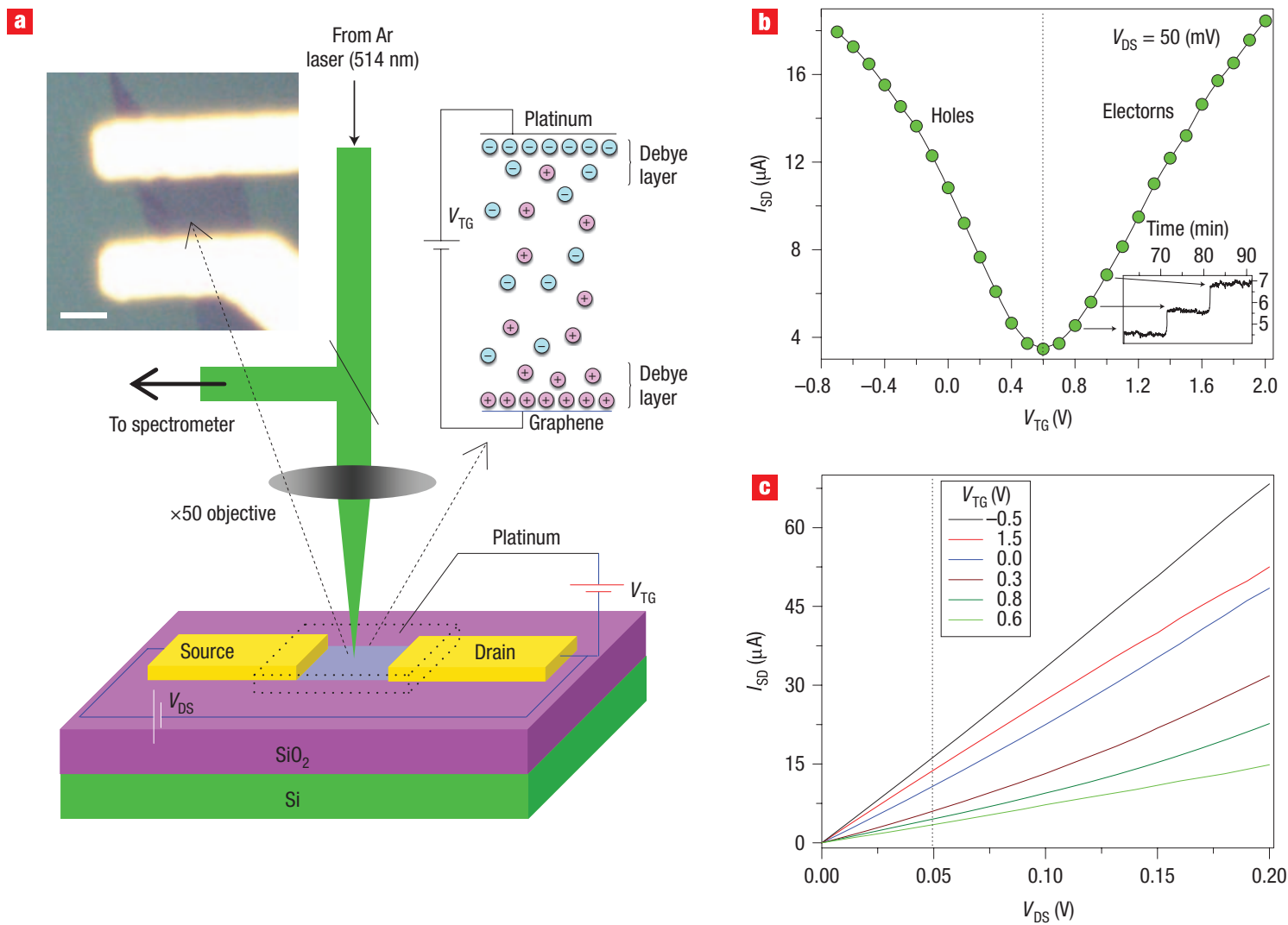

Figure 1 Electrochemically top-gated graphene transistor. a, Schematic diagram of the experimental setup. The black dotted box between the drain and source indicates the thin layer of polymer electrolyte $\left(\mathrm{PEO}+\mathrm{LiClO}_{4}\right)$, and the blue stripe between the electrodes represents the graphene sample. The left inset shows the optical image of a single-layer graphene connected between source and drain gold electrodes. Scale bar: $5 \mu \mathrm{m}$. The right inset is a schematic illustration of polymer electrolyte top gating, with $\mathrm{Li}^{+}$(magenta) and $\mathrm{ClO}_{4}^{-}$(cyan) ions and the Debye layers near each electrode. $\mathbf{b}, I_{\mathrm{SD}}$ as a function of top-gate voltages $\left(V_{\mathrm{TG}}\right)$. The inset shows the $I_{\mathrm{SD}}$ time dependence at fixed $V_{\mathrm{TG}}$. The dotted line corresponds to the Dirac point (change neutrality point). c, $I_{\mathrm{SD}}$ versus $V_{\mathrm{DS}}$ at different top-gate voltages. The black dotted line corresponds to the value of $V_{D S}$ at which the data in Fig. $1 \mathrm{~b}$ was measured.

sample. Figure 2a,b also show that, for both top-gate (TG) and backgate (BG) experiments, the resistivity does not decay sharply around the Dirac point. Indeed, it has been suggested that the sharpness of the resistivity around the Dirac point and the finite offset gate voltage $\left(V_{n \mathrm{BG}}\right)$ depend on charged impurities ${ }^{12}$.

The conductivity minimum $\left(\sigma_{\min }\right)$ (resistivity maximum) is obtained when the Fermi level is at the Dirac point. This is generally around $\sim 4 e^{2} / h$ (ref. 2 ). In both our back- and top-gate experiments the conductivity minimum is reduced by the contact resistance, because measurements are performed in the twoprobe configuration, or possible contaminations at the contactgraphene interface. Minimum conductivities in the range from $2 e^{2} / h$ to $10 e^{2} / h$ have been reported recently ${ }^{12}$, with the spread assigned to charged impurities.

Figure $2 \mathrm{c}$ shows the change in mobility (using the simple Drude model $\left.^{12} \mu=(e n \rho)^{-1}\right)$ as a function of doping for our TG/BG experiments. The Drude model can be safely used here, because the sample length $(\sim 5 \mu \mathrm{m})$ is much more than the transport mean free path $(\sim 100 \mathrm{~nm})^{11-13}$. The mobility is smaller in the TG case. This is consistent with the reduction in conductivity minimum and can be attributed to the presence of added charge impurities from the polymer electrolyte. This reduction in mobility for TG is consistent with ref. 4 .

Despite the limitations in 'on' and 'off' currents, our large graphene device shows an on/off ratio of $\sim 5.5$. This is higher than previously reported results ${ }^{4}$ for devices using 20 -nm-thick $\mathrm{SiO}_{2}$ as a top gate (on/off ratio $\sim 1.5$ ) and 40 -nm-thick PMMA ${ }^{14}$ as a top gate (on/off ratio $\sim 2$ ). Our demonstration of top gating with polymer electrolyte paves the way for further research. For example, by using water as the top gate and extensive graphene cleaning we could achieve an on-off ratio of 40 (see Supplementary Information). However, because the water droplet evaporates in less than one minute, this arrangement is not stable over long periods of time, unlike the solid polymer electrolyte.

Raman spectroscopy is a powerful non-destructive technique for identifying the number of layers, structure, doping and disorder of graphene $e^{15-19}$. The prominent Raman features in graphene are the G-band at $\Gamma\left(\sim 1,584 \mathrm{~cm}^{-1}\right)$, and the $2 \mathrm{D}$ band at $\sim 2,700 \mathrm{~cm}^{-1}$ involving phonons at the $\mathbf{K}+\Delta \mathbf{k}$ points in the brillouin zone ${ }^{15}$. The value of $\Delta \mathbf{k}$ depends on the excitation laser energy, due to a double-resonance Raman process and the linear dispersion of the phonons around $\mathbf{K}$ (refs. 15, 20, 21). The effect of doping induced by $\mathrm{SiO}_{2}$ back gating on the G-band frequency and full-width at half-maximum (FWHM) has been reported recently ${ }^{16,17}$. This results in $G$ peak stiffening and a decrease in linewidth for both electron and hole doping. The decrease in linewidth saturates when the doping causes a Fermi-level shift bigger than half the phonon energy ${ }^{16,17}$. The strong electronphonon coupling in graphene and metallic nanotubes gives rise to Kohn anomalies in the phonon dispersions ${ }^{21-23}$, which result 

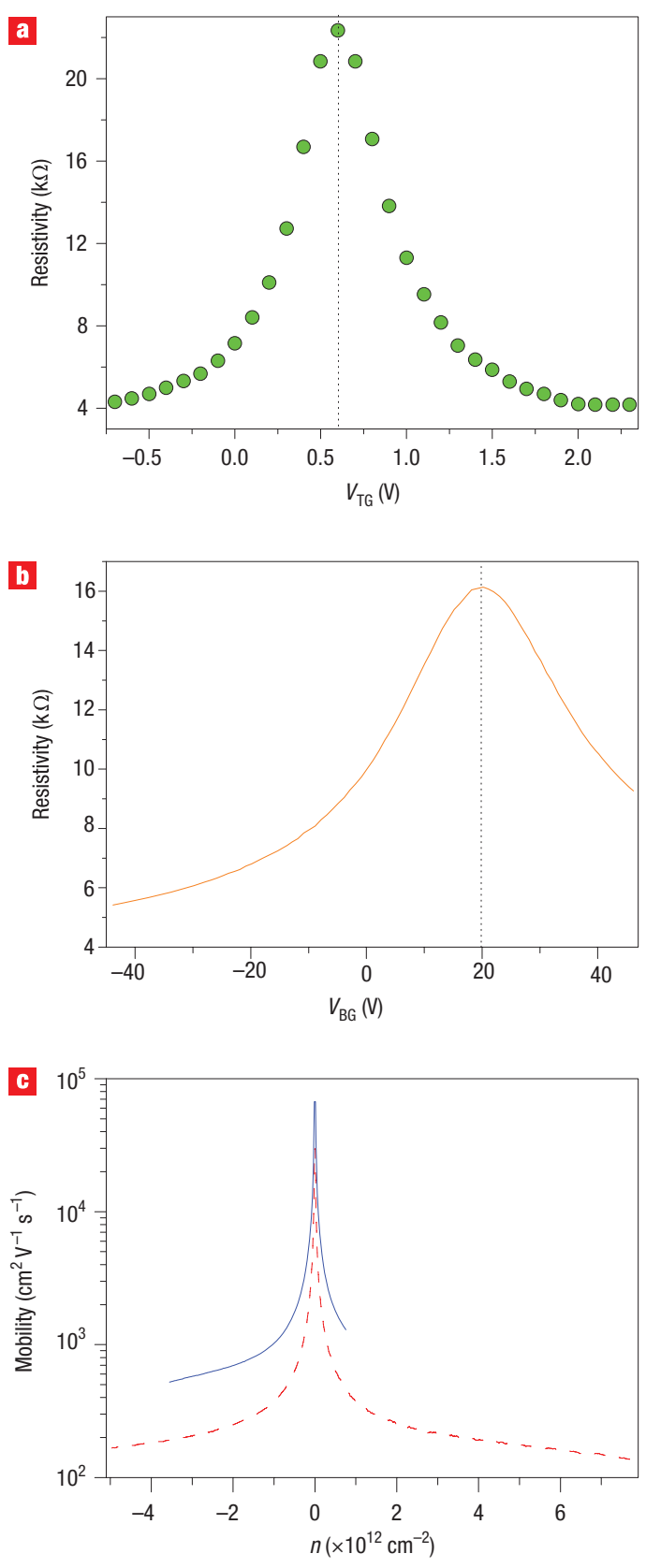

Figure 2 Conductivity minimum in graphene. a, Resistivity as a function of the top-gate voltage. The dots are extracted from Fig. $1 \mathrm{~b}$ for $W / L=1.55$. b, Resistivity of the same sample as a function of the back-gate voltage. The dotted black line marks the Dirac point. c, Mobility as a function of doping for top gating (dashed red line) and back gating (solid blue line).

in phonon softening. The G peak stiffening is due to the nonadiabatic removal of the Kohn anomaly from the $\Gamma$ point ${ }^{16}$. The FWHM(G) sharpening occurs because of the blockage of the decay channel of phonons into electron-hole pairs due to the Pauli exclusion principle, when the electron-hole gap becomes higher than the phonon energy ${ }^{16}$. A similar behaviour is observed for the longitudinal optic (LO) $\mathrm{G}^{-}$peak of doped metallic nanotubes ${ }^{8,9,24}$, for exactly the same reasons.

We now consider the evolution of the Raman spectra. Figure $3 \mathrm{a}$ plots the Raman spectra in the G (left) and $2 \mathrm{D}$ (right) region at different values of the top-gate voltage. Figure 3b,c shows how the Raman parameters (the positions of the $\mathrm{G}$ and $2 \mathrm{D}$ peaks, and the FWHM of the G peak) vary as a function of doping. The Raman shift of the $G$ peak has its smallest value $\left(\sim 1,583.1 \mathrm{~cm}^{-1}\right)$ at $V_{\mathrm{TG}}=V_{n \mathrm{TG}} \sim 0.6 \mathrm{~V}$, and increases by up to $30 \mathrm{~cm}^{-1}$ for hole doping and up to $25 \mathrm{~cm}^{-1}$ for electron doping (Fig. 3b, top panel). The decrease in the FWHM of the G peak (Fig. 3b, bottom panel) for both hole and electron doping is similar to earlier results ${ }^{16,17}$, even though it extends to a much wider doping range. Moreover, the $2 \mathrm{D}$ and $\mathrm{G}$ peak show very different dependencies on the gate voltage. For electron doping, the position of the $2 \mathrm{D}$ peak does not change much $\left(<1 \mathrm{~cm}^{-1}\right)$ until a gate voltage of $\sim 3 \mathrm{~V}$ (corresponding to $\sim 3.2 \times 10^{13} \mathrm{~cm}^{-2}$ ). At higher gate voltages, there is a significant softening of $\sim 20 \mathrm{~cm}^{-1}$ and for hole doping, the position of the $2 \mathrm{D}$ peak increases $\sim 20 \mathrm{~cm}^{-1}$ (Fig. $3 \mathrm{c}$ ).

Figure 4 plots the variation of the intensity ratio of the $G$ and $2 \mathrm{D}$ peaks $(I(2 \mathrm{D}) / I(\mathrm{G}))$ as a function of doping. The dependence of the $2 \mathrm{D}$ mode is much stronger than that of the $\mathrm{G}$ mode and hence $I(2 \mathrm{D}) / I(\mathrm{G})$ is a strong function of the gate voltage. Therefore, this is a new, important parameter to estimate the doping density. Figures 3 and 4 also show that $I(2 \mathrm{D}) / I(\mathrm{G})$ and the position of the $G$ peak should not be used to estimate the number of graphene layers, contrary to what is suggested in refs 25 and 26. It is the shape of the $2 \mathrm{D}$ peak that is the most effective way to identify a single layer, as shown in ref. 15.

The theoretical trends in Fig. $3 b$ have been discussed before ${ }^{16}$. These confirm previous back-gate experiments, but extend the data to a much wider electron and hole range ${ }^{16}$. In this wider range, the theory still captures the main features, such as the asymmetry between electron and hole doping ${ }^{27}$. However, the quantitative agreement is poor for large doping, and requires us to reconsider the non-adiabatic calculations of ref. 27. At low doping, the uncertainty, as estimated by comparing the Raman data and theory, is at most $25 \%$.

Here we focus on the novel trend of the $2 \mathrm{D}$ peak position as a function of doping. This is experimentally and conceptually different from the interpretation of the $G$ peak. The 2D peak originates from a second-order, double-resonant (DR) Raman scattering mechanism ${ }^{15,20,28}$. The position of the $2 \mathrm{D}$ peak can be evaluated by computing the energy of the phonons involved in the second-order, DR scattering process. As shown in ref. 15, because of the trigonal warping of the $\pi-\pi^{\star}$ bands and the angular dependence of the electron-phonon coupling (EPC) matrix elements, only phonons oriented along the ГKM direction and with $\mathbf{q}>\mathbf{K}$ give a non-negligible contribution to the $2 \mathrm{D}$ peak. The precise value of $q$ is fixed by the constraint that the energy of the incoming laser photons $\left(\hbar \omega_{\mathrm{L}}\right)$ has to exactly match a real electronic transition. In particular, only a wavevector $\mathbf{q}^{\prime}$ can be found for which $\hbar \omega_{\mathrm{L}}=\epsilon\left(\pi^{*}, \mathbf{q}^{\prime}\right)-\epsilon\left(\pi, \mathbf{q}^{\prime}\right)$, where $\boldsymbol{\epsilon}(n, \mathbf{k})$ is the energy of an electron of band index $n$ and wavevector $\mathbf{k}$, and $\mathbf{q}^{\prime}$ is measured from $\mathbf{K}$ and is in the $\mathbf{\Gamma} \mathbf{K M}$ direction. Once $\mathbf{q}^{\prime}$ has been determined, $q=2 q^{\prime}+K$. Among the six phonons corresponding to the $\mathbf{q}$ vector that satisfy the DR conditions, only the highest optical branch has an energy compatible with the measured Raman shift. Therefore, the theoretical position of the 2D peak corresponds to twice the energy of the Raman active phonon.

In order to be comparable with our experiments performed at $514 \mathrm{~nm}$, we consider $\hbar \omega_{\mathrm{L}}=2.5 \mathrm{eV}$. Assuming the $\pi / \pi^{\star}$ bands to be linear, with a slope of $14.1 \mathrm{eV}$ (ref. 21), this laser energy selects a phonon with wavevector $\mathbf{q}$ of modulus 0.844 in $2 \pi / a_{0}$ units, where $a_{0}$ is the lattice parameter of graphene. The dependence of the position of the $2 \mathrm{D}$ peak on doping can be investigated by calculating, within a density functional theory (DFT) framework, the effects of the Fermi-level shift on the phonon frequencies. 

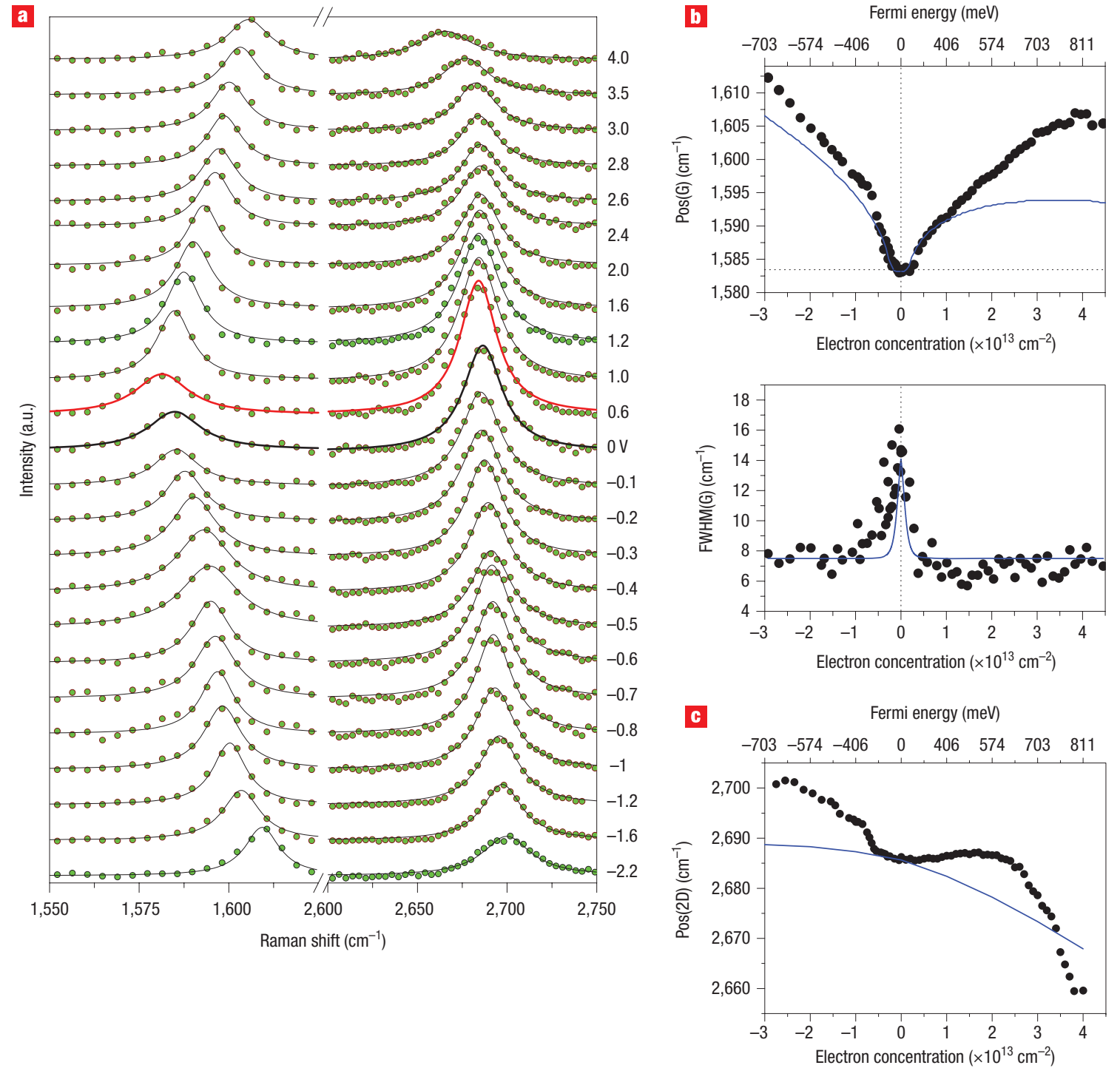

Figure 3 Raman spectra of graphene as a function of gate voltage. a, Raman spectra at values of $V_{T G}$ between $-2.2 \mathrm{~V}$ and $+4.0 \mathrm{~V}$. The dots are the experimental data, the black lines are fitted lorentzians, and the red line corresponds to the Dirac point. The G peak is on the left and the 2D peak is on the right. b, Position of the G peak (Pos(G)); top panel) and its FWHM (FWHM(G); bottom panel) as a function of electron and hole doping. The solid blue lines are the predicted non-adiabatic trends from ref. 16. c, Position of the $2 \mathrm{D}$ peak (Pos(2D)) as a function of doping. The solid line is our adiabatic DFT calculation.

In doped graphene, the shift of the Fermi energy induced by doping has two major effects: (1) a change of the equilibrium lattice parameter with a consequent stiffening/softening of the phonons, and (2) the onset of effects beyond the adiabatic Born-Oppenheimer approximation that modify the phonon dispersion close to the Kohn anomalies (KAs) ${ }^{16,27}$. The excess (defect) charge results in an expansion (contraction) of the crystal lattice. This has been extensively investigated in order to understand graphite intercalation compounds ${ }^{29}$. We model the shift of the Fermi surface by varying the number of electrons in the system. Because the total energy of charged systems diverges, electrical neutrality is achieved by imposing a uniformly charged background. To avoid electrostatic interactions between the graphene layer and the background, the equilibrium lattice parameter of the charged systems is computed in the limit of a unit cell with an infinite volume. Such a limit is reached by using a model with periodic boundary conditions where the graphene layers are spaced by $60 \AA$ vacuum. Phonon calculations for charged graphene are carried out with the same unit cells used for the determination of the corresponding lattice parameter. Interestingly, although we observe that for charged graphene the frequency of border zone phonons converges only for layer spacing as large as $60 \AA$, the frequency of the $E_{2 g}$ mode is already converged for a $7.5 \AA$ spacing. This suggests that border zone phonons are much more sensitive to the local environment.

Dynamic effects beyond the Born-Oppenheimer approximation play a fundamental role in the description of the KA in single-walled carbon nanotubes and in graphene ${ }^{16,22,27}$. However, for the 2D peak measured at $514 \mathrm{~nm}$, the influence of dynamic effects is expected to be negligible, because the phonons giving rise to the $2 \mathrm{D}$ peak are far away from the $\mathrm{KA}$ at $\mathbf{K}$. Thus, we can calculate the position of the $2 \mathrm{D}$ peak without dynamic corrections (see Methods). 


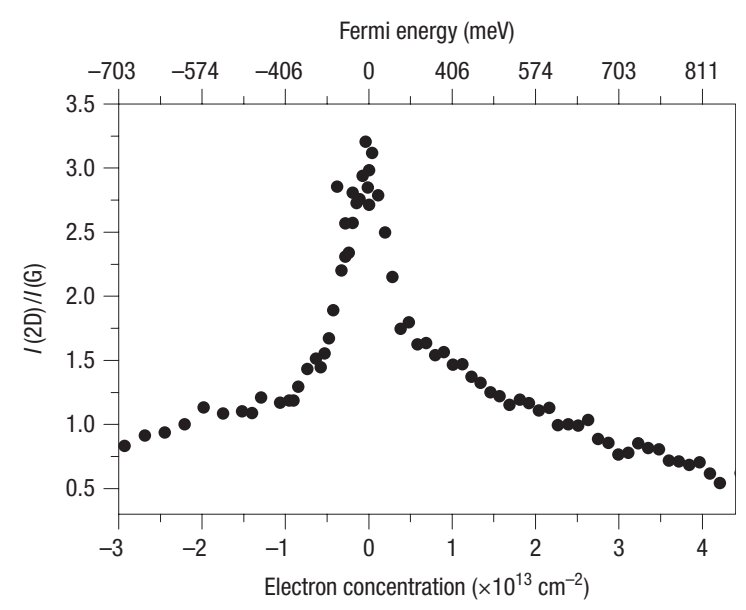

Figure 4 The influence of hole and electron doping on the $2 \mathrm{D}$ and $\mathrm{G}$ peaks. The ratio of the intensity of the $2 \mathrm{D}$ peak in the Raman spectrum to the intensity of the $\mathrm{G}$ peak exhibits a clear dependence on the electron concentration, and can therefore be used to monitor the level of doping in graphene-based devices.

The comparison between the theoretical and the experimental position of the 2D peak is shown in Fig. 3c. Our calculations are in qualitative agreement with experiments, considering the spectral resolution and the Debye layer estimation. Indeed, as experimentally determined, the position of the $2 \mathrm{D}$ peak is predicted to decrease for an increasing electron concentration in the system. This allows the use of the $2 \mathrm{D}$ peak to discriminate between electron and hole doping.

The tradeoff between measured and theoretical data can be partially explained in terms of the electrostatic difference existing between the experiments and the model DFT system. In our simulations, the $2 \mathrm{D}$ phonon frequencies are very sensitive to the charged background used to ensure global electrical neutrality. In the experiments, the electric charge on the graphene surface is induced by capacitative coupling. The electrostatic interaction between graphene and the electrolyte could thus further modify the $2 \mathrm{D}$ phonons. This does not affect the $\mathrm{G}$ peak to the same extent, due to the much lower sensitivity of the G phonon to an external electrostatic potential. Other effects not captured by DFT, such as quasi-particle interactions, should also be considered to fully explain the 2D peak behaviour.

In conclusion, we have demonstrated the first graphene top gating using a solid polymer electrolyte. We reached much higher electron and hole doping than standard $\mathrm{SiO}_{2}$ back gating. The Raman measurements show that the $G$ and $2 \mathrm{D}$ peaks have different doping dependence and the $2 \mathrm{D} / \mathrm{G}$ height ratio changes significantly with doping, making Raman spectroscopy an ideal tool for graphene nanoelectronics.

\section{METHODS}

\section{EXPERIMENTAL}

Graphene samples were produced by micro-mechanical cleavage of bulk graphite and deposited on Si covered with 300-nm $\mathrm{SiO}_{2}$ (IDB Technologies). Raman spectroscopy was used to select single layers ${ }^{15}$. Source and drain $\mathrm{Cr} / \mathrm{Au}$ electrodes were then deposited by photolithography as shown in Fig. 1a. Cr was used instead of $\mathrm{Ti}$ to ensure less reactivity with the electrolyte. Top gating was achieved by using solid polymer electrolyte consisting of $\mathrm{LiClO}_{4}$ and PEO in the ratio $0.12: 1$, as previously used for nanotubes ${ }^{10}$. The gate voltage was applied by placing a platinum electrode in the polymer layer ${ }^{10}$. Electrical measurements were carried out using Keithley 2400 source meters. Figure 1 shows a schematic of the experimental setup for transport and Raman measurements. Raman spectra of pristine and back-gated samples were measured with a Renishaw spectrometer. In situ measurements on top-gated graphene were recorded using a WITEC confocal $(\times 50$ objective $)$ spectrometer with 600 lines $/ \mathrm{mm}$ grating, $514.5 \mathrm{~nm}$ excitation and very low power level $(\sim 1 \mathrm{~mW})$ to avoid any heating effect. The spectral resolution of the two instruments was determined by fitting the Rayleigh line to a gaussian profile and is $1.9 \mathrm{~cm}^{-1}$ for the Renishaw spectrometer and $9.4 \mathrm{~cm}^{-1}$ for the WITEC spectrometer. The Raman spectra were then fitted with Voigt functions. The FWHM of the lorentzian components give the relevant information on the phonon lifetime. Note that a very thin layer of polymer electrolyte does not absorb the incident laser light. Furthermore, the Raman spectrum of the polymer does not cover the signatures of graphene (see Supplementary Information). The measured source-drain currents $\left(I_{\mathrm{SD}}\right)$ and $\mathrm{G}$ and $2 \mathrm{D}$ are reversible at different gate voltages. Note that for each point a given gate voltage is applied for $10 \mathrm{~min}$ to stabilize $I_{\mathrm{SD}}$. In transport experiments a small hysteresis in current $(\sim 1 \mu \mathrm{A})$ is observed during forward and backward gate voltage scans (at intervals of $10 \mathrm{~min}$ for each gate-voltage step). The Raman hysteresis, however, is less than $1 \mathrm{~cm}^{-1}$.

\section{GATE VOLTAGES AND DOPING LEVELS}

We now discuss how the applied gate voltage is converted to the doping in graphene. Let us first consider back gating. For a back gate, $\phi=n e / C_{\mathrm{BG}}$, where $n$ is the carrier concentration and $C_{\mathrm{BG}}$ is the geometrical capacitance. For single-layer graphene, $C_{\mathrm{BG}}=\epsilon \epsilon_{0} / d_{\mathrm{BG}}$, where $\epsilon$ is the dielectric constant of $\mathrm{SiO}_{2}$ $(\sim 4), \epsilon_{0}$ is the permittivity of free space and $d_{\mathrm{BG}}$ is $300 \mathrm{~nm}$. This results in a very low gate capacitance $C_{\mathrm{BG}}=1.2 \times 10^{-8} \mathrm{~F} \mathrm{~cm}^{-2}$. Therefore, for a typical value of $n=1 \times 10^{13} \mathrm{~cm}^{-2}$, the potential drop is $\phi=100 \mathrm{~V}$, much larger than $E_{\mathrm{F}} / e$. Hence, $V_{\mathrm{BG}} \approx \phi$ and the doping concentration becomes $n=\eta V_{\mathrm{BG}}$, where $\eta=C_{\mathrm{BG}} / e$. However, most samples have a zero-bias $\left(V_{\mathrm{BG}}=0\right)$ doping of, typically, a few $10^{11} \mathrm{~cm}^{-2}$ (refs $\left.1,18,19\right)$. This is reflected in the existence of a finite gate voltage $V_{n \mathrm{BG}}$, at which the Hall resistance is zero and the longitudinal resistivity reaches its maximum. This maximum is associated with the Fermi level positioned between the valence and the conduction bands (the Dirac point). Accordingly, a positive (negative) $V_{\mathrm{BG}}-V_{n \mathrm{BG}}$ induces electron (holes) doping, with an excess electron surface-concentration of $n=\eta\left(V_{\mathrm{BG}}-V_{n \mathrm{BG}}\right)$. A value of $\eta \approx 7.2 \times 10^{10} \mathrm{~cm}^{-2} V^{-1}$ is found from Hall effect measurements, and agrees with the estimation from the gate geometry ${ }^{1-3}$.

We shall now consider the present case of top gating. When a field is applied, free cations tend to accumulate near the negative electrode, creating a positive charge there and an uncompensated negative charge near the interface. The accumulation is limited by the concentration gradient, which opposes the Coulombic force of the electric field. When a steady state is reached, the statistical space charge distribution resembles that shown in Fig. 1. This layer of charge around an electrode is called the Debye layer. As shown in Fig. 1, when we apply a positive potential $\left(V_{\mathrm{TG}}\right.$ ) to the platinum top gate (with respect to the source electrode connected to graphene), the $\mathrm{Li}^{+}$ions become dominant in the Debye layer formed at the interface between the graphene and the electrolyte. The Debye layer of thickness $d_{\mathrm{TG}}$ acts like a parallel-plate capacitor. Therefore, the geometrical capacitance in this case is $C_{\mathrm{TG}}=\epsilon \epsilon_{0} / d_{\mathrm{TG}}$, where $\epsilon$ is the dielectric constant of the PEO matrix. The Debye length is given by $d_{\mathrm{TG}}=\left(2 c e^{2} / \epsilon \epsilon_{0} k T\right)^{-1 / 2}$ for a monovalent electrolyte, where $c$ is the concentration of the electrolyte, $e$ is the electric charge and $k T$ is the thermal energy. In principle, $d_{\mathrm{TG}}$ can be calculated if the electrolyte concentration is known. However, in the presence of a polymer, the electrolyte ions form complexes with the polymer chains ${ }^{30}$. Hence, the exact concentration of ions is not amenable to measurement. For polymer electrolyte gating the thickness of the Debye layer is reported to be a few nanometres $(\sim 1-5 \mathrm{~nm})$ (ref. 10). The dielectric constant $\epsilon$ of PEO is 5 (ref. 31). Assuming a Debye length of $2 \mathrm{~nm}$, we obtain a gate capacitance $C_{\mathrm{TG}}=2.2 \times 10^{-6} \mathrm{~F} \mathrm{~cm}^{-2}$, which is much higher than $C_{\mathrm{BG}}$. Therefore, the first term in equation (1) cannot be neglected.

THEORY

Calculations were performed within the generalized gradient approximation $(\text { GGA })^{32}$. We used planewaves (30 Ry cutoff) and pseudopotential approaches. The semimetallic character of the system was treated by performing electronic integration with a Fermi-Dirac first-order spreading with a smearing of 0.01 Ry. Integration over the brillouin zone was covered out with a uniform $72 \times 72 \times 1 k$-points grid. Calculations were carried out using the Quantum Espresso code (www.quantum-espresso.org).

Received 30 October 2007; accepted 28 February 2008; published 30 March 2008. 


\section{References}

1. Novoselov, K. S. et al. Electric field effect in atomically thin carbon films. Science $\mathbf{3 0 6}$ $666-669$ (2004)

2. Novoselov, K. S. et al. Two-dimensional gas of massless Dirac fermions in graphene. Nature 438, 197-200 (2005).

3. Zhang, Y., Tan, Y.-W., Stormer, H. L. \& Kim, P. Experimental observation of the quantum Hall effect and Berrýs phase in graphene. Nature 438, 201-204 (2005).

4. Lemme, M.C., Echtermeyer, T.J., Baus, M. \& Kurz, H. A Graphene field-effect device. IEEE Electron. Device Lett. 28, 282-284 (2007).

5. Han, M. Y., Ozyilmaz, B., Zhang, Y. \& Kim, P. Energy band-gap engineering of graphene nanoribbons. Phys. Rev. Lett. 98, 206805 (2007).

6. Sirringhaus, H. et al. High-resolution inkjet printing of all-polymer transistor circuits. Science 290, 2123-2126 (2000).

7. Dhoot, A. S. et al. Beyond the metal-insulator transition in polymer electrolyte gated polymer field-effect transistors. Proc. Natl Acad. Sci. USA 103, 11834-11837 (2006).

8. Nguyen, K. T., Gaur, A. \& Shim, M. Fano lineshape and phonon softening in single isolated metallic carbon nanotubes. Phys. Rev. Lett. 98, 145504 (2007).

9. Das, A. et al. Doping in carbon nanotubes probed by raman and transport measurements. Phys. Phys. Lett. 99, 136803 (2007).

10. Lu, C., Fu, Q., Huang, S. \& Liu, J. Polymer electrolyte-gated carbon nanotube field-effect transistor Nano Lett. 4, 623-627 (2004).

11. Geim, A. K. \& Novoselov, K. S. The rise of graphene. Nature Mater. 6, 183-191 (2007).

12. Tan, Y.-W. et al. Measurement of scattering rate and minimum conductivity in graphene. Phys. Rev. Lett. 99, 246803 (2007).

13. Morozov, S. V. et al. Giant intrinsic carrier mobilities in graphene and its bilayer. Phys. Rev. Lett. 100 016602 (2008)

14. Huard, B. et al. Transport measurements across a tunable potential barrier in graphene. Phys. Rev. Lett. 98, 236803 (2007).

15. Ferrari, A. C. et al. Raman spectrum of graphene and graphene layers. Phys. Rev. Lett. 97, 187401 (2006).

16. Pisana, S. et al. Breakdown of the adiabatic Born-Oppenheimer approximation in graphene. Nature Mater. 6, 198-201 (2007)

17. Yan, J., Zhang, Y., Kim, P. \& Pinczuk, A. Electric field effect tuning of electron-phonon coupling in graphene. Phys. Rev. Lett. 98, 166802 (2007).

18. Casiraghi, C., Pisana, S., Novoselov K. S., Geim A. K. \& Ferrari A. C. Raman fingerprint of charged impurities in graphene. Appl. Phys. Lett. 91, 233108 (2007).

19. Stampfer, C. et al. Raman imaging of charged domains in graphene on $\mathrm{SiO}_{2}$. Appl. Phys. Lett. 91 , 241907 (2007).
20. Thomsen, C. \& Reich, S. Double resonant Raman scattering in graphite. Phys. Rev. Lett. 85, 5214-5217 (2000)

21. Piscanec, S., Lazzeri, M., Mauri, F., Ferrari, A. \& Robertson, J. Kohn anomalies and electron-phonon interactions in graphite. Phys. Rev. Lett. 93, 185503 (2004).

22. Piscanec, S., Lazzeri, M., Robertson, J., Ferrari, A. C. \& Mauri, F. Optical phonons in carbon nanotubes: Kohn anomalies, Peierls distortions, and dynamic effects. Phys. Rev. B 75, 035427 (2007).

23. Lazzeri, M., Piscanec, S., Mauri, F., Ferrari, A. C. \& Robertson, J. Phonon linewidths and electronphonon coupling in graphite and nanotubes. Phys. Rev. B 73, 155426 (2006).

24. Tsang, J. C., Freitag, M., Perebeinos, V., Liu, J. \& Avouris, P. H. Doping and phonon renormalization in carbon nanotubes. Nature Nanotech. 2, 725-730 (2007).

25. Gupta, A., Chen, G., Joshi, P., Tadigadapa, S. \& Eklund, P. C. Raman scattering from high-frequency phonons in supported $n$-graphene layer films. Nano Lett. 6, 2667-2673 (2006).

26. Graf, D. et al. Spatially resolved Raman spectroscopy of single- and few-layer graphene. Nano Lett. 7 , $238-242(2007)$

27. Lazzeri, M. \& Mauri, F. Nonadiabatic Kohn anomaly in a doped graphene monolayer. Phys. Rev. Lett. 97, 266407 (2006)

28. Maultzsch, J., Reich, S. \& Thomsen, C. Chirality-selective Raman scattering of the D mode in carbon nanotubes. Phys. Rev. B 61, 121407 (2001)

29. Pietronero, L. \& Strassler, S. Bond-length change as a tool to determine charge transfer and electronphonon coupling in graphite intercalation compounds. Phys. Rev. Lett. 47, 593-596 (1981)

30. Salomon, M., Xu, M., Eyring, E. M. \& Petrucci, S. Molecular structure and dynamics of LiC104-polyethylene oxide-400 (dimethyl ether and diglycol systems) at $25^{\circ}$ C. J. Phys. Chem. 98 , 8234-8244 (1994).

31. Boyd, R. H. The dielectric constant of lamellar semicrystalline polymers. J. Polym. Sci. Polym. Phys. Ed. 21, 505-514 (1983).

32. Perdew, P., Burke, K. \& Ernzerhof, M. Generalized gradient approximation made simple. Phys. Rev. Lett. 77, 3865-3868 (1996).

Acknowledgements

S.P. acknowledges funding from Pembroke College and the Maudslay Society. A.C.F. acknowledges funding from the Royal Society and Leverhulme Trust. A.K.S. thanks the Department of Science and Technology, India, for financial support.

Correspondence and requests for materials should be addressed to A.C.F. and A.K.S.

Supplementary Information accompanies this paper on www.nature.com/naturenanotechnology.

Reprints and permission information is available online at http://npg.nature.com/reprintsandpermissions/ 